\title{
Double-Jump Migration and Diffusive Instability
}

\author{
YUNXIN HUANG* AND ODO DIEKMANN
}

Mathematical Department, Utrecht University,

P.O. Box 80010,

3508 TA Utrecht,

The Netherlands

\section{FRANK VAN DEN BOSCH}

IACR-Rothamsted,

Harpenden, Hert AL5 2JQ,

UK

In order to look into the stability consequences of a particular migration process in which individuals choose to settle, we formulated a continuous-time multi-species multi-patch model in which individuals migrate by one or two instantaneous jumps while making the second jump with a certain probability that possibly depends on the conditions at the end point of the first jump. It turned out that a second jump has some quantitative effects on diffusive instability even when it occurs in the absence of density-dependent mechanisms. When a second jump happens as a natural interspecific response of individuals, and such a response is sufficiently strong, it has crucial effects on diffusive instability: it leads to diffusive instability in the case of competitive interactions, whereas it annihilates diffusive instability in the case of prey-predator interactions. We demonstrated these results in the context of two specific examples.

(C) 2003 Society for Mathematical Biology. Published by Elsevier Ltd. All rights reserved.

\section{INTRODUCTION}

One of the major issues in spatial population ecology is how dispersal combined with population dynamics influences stability and persistence of interacting species. Although a tremendous amount of theoretical work has been devoted to investigating the issue [e.g., Turing (1952), Okubo (1980), Holt (1984, 1985), Segel (1984), Karevia and Odell (1987), Othmer et al. (1988), Okubo and Levin (2001) etc.], the underlying mechanisms are far from being completely understood because of the complexity of dispersal processes.

${ }^{*}$ Corresponding address: Faculty of Mathematics and Computer Science, Hubei University, Wuhan 430062, P. R. China.E-mail: yunxin@public.wh.hb.cn or y.huang@hubu.edu.cn

$0092-8240 / 04 / 030487+18 \quad \$ 30.00 / 0$

Elsevier Ltd. All rights reserved.

(C) 2003 Society for Mathematical Biology. Published by 
In a general model the following aspects of migration could be taken into account

(i) the trigger for emigration

(ii) the movement itself

(iii) assessment of the 'landing' position.

In an earlier paper (Huang and Diekmann, 2003) we focussed on the first aspect (i) and, in particular, on the consequences for pattern formation of triggers produced by (the density of) another species. [In the case of predator-prey interactions, for instance, prey at a certain position may have increasing inclination to leave when the number of predators near-by increases because of the increasing danger, whereas predators, in contrast, may have decreasing inclination to emigrate when the number of prey increases because of the increasing food abundance (Huang and Diekmann, 2001).] In the present paper we intend, in a similar spirit, to investigate the consequences for pattern formation of the third aspect (iii). More specifically, we are going to formulate and analyse a model in which migrating individuals who 'land' for the first time have two options: to settle or to depart for another journey. For example, predatory mites dispersing in the air usually look for a 'good place' to settle, while the settlement of free swimming larvae of some rock-shore species is often inhibited by chemical defence mechanisms of competitors. Even bird and mammal species preferentially settle at places of higher prey density. In short, after travelling to a place, individuals may 'choose' to settle or to travel again immediately, depending on the 'quality' of the place. In both papers we adopt a caricatural description of the movement itself: it takes no time, no cost (no deaths occur), and it is completely random in the sense that, given a starting patch, the distribution of landing patches reflects only the geographical structure and is independent of any densities (so, in particular, we do not incorporate directed movement as in chemotaxis). Also concerning the third aspect (iii) we make simplifying assumptions: the assessment takes no time, no cost. Our aim is not to describe any specific migrating organism in some detail. Instead our aim is to point out that, apart from chemotaxis, there are several ways in which interspecific influence on mobility may show up in spatial population models (Skellam, 1973; Aronson, 1985). In the previous paper (Huang and Diekmann, 2003) we focussed on the start of a migration event whereas we concentrate on the end of such a event in the present paper.

To model such a migration process by a continuous-time multi-patch model, we need to extend our previous formulation. Indeed, the dispersal process described in the last paragraph may be regarded as consisting of two or more jumps. The approach to dividing a spatio-temporal transition process into consecutive subprocesses (e.g., a sedentary stage of growth and interaction and a mobile stage) has been used in formulating discrete-time spatial models (Kot, 1992; Neubert et al., 1995; Lewis and Pacala, 2000; Weinberger et al., 2002). There are also some early literatures in the context of spatial spread of infectious diseases (Mollison, 1977; Diekmann, 1978, 1979; Thieme, 1979a,b). In the case where individuals do move 
by jumps of short duration (Othmer et al., 1988), we may use this method to formulate a continuous-time model.

In this paper, we shall indeed formulate a continuous-time multi-species multipatch model in which migration of individuals of each species is in general a composite of two instantaneous jumps. We shall assume that there is a probability for individuals to make a second jump (which depends in general on the conditions at the end point of the first jump). We shall first examine if and how the second jump, when it is assumed to be density-independent (so merely featuring the approach of double-jump scheme of migration), changes the conditions for diffusive instability as compared to the standard approach. We then study whether or not the second jump, when it happens as a natural interspecific response of individuals, affects diffusive instability crucially.

\section{THE MODEL}

Consider a system of $m$ species in a habitat consisting of $n$ identical patches. Suppose that individuals of each species migrate by way of instantaneous jumps (we shall restrict the number of jumps in one migration event to at most two in order to arrive at a manageable formulation). For any species $i$ in any patch $j$, the rate of density change matches the sum of the net growth rate and the net migration rate (i.e., immigration minus emigration), yielding the following system of ordinary differential equations:

$$
\begin{gathered}
\frac{d u_{j}}{d t}=f\left(u_{j}\right)-M u_{j}+\left(I-\Phi\left(u_{j}\right)\right) \sum_{k=1}^{n} q_{k j} M u_{k}+\sum_{l=1}^{n} q_{l j} \Phi\left(u_{l}\right) \sum_{k=1}^{n} q_{k l} M u_{k} \\
j=1,2, \ldots, n
\end{gathered}
$$

in which $u_{j}=\left(u_{1 j}, \ldots, u_{m j}\right)^{T} \in \mathbb{R}^{m}$ is an $m$-vector whose element $u_{i j}$ is the density of species $i$ in patch $j$, while

- $f(\cdot)=\left(f_{1}(\cdot), \ldots, f_{m}(\cdot)\right)^{T}: \mathbb{R}^{m} \rightarrow \mathbb{R}^{m}$ is a vector function in which $f_{i}(\cdot)$ $(i=1, \ldots, m)$ is the local growth rate of species $i$.

- $M=\operatorname{diag}\left\{m_{1}, \ldots, m_{m}\right\}: \mathbb{R}^{m} \rightarrow \mathbb{R}^{m \times m}$ in which $m_{i}(i=1, \ldots, m)$ is the per capita emigration rate of species $i$ (from any patch). $m_{i}$ may be densitydependent in general [which is in fact the case we have studied in the previous work (Huang and Diekmann, 2003)], but we assume that $m_{i}$ is densityindependent in this paper in order to focus on our main concerns.

- $\Phi(\cdot)=\operatorname{diag}\left\{\phi_{1}(\cdot), \ldots, \phi_{m}(\cdot)\right\}: \mathbb{R}^{m} \rightarrow \mathbb{R}^{m \times m}$ in which $\phi_{i}(\cdot)(i=1, \ldots, m)$ is the probability that an individual of species $i$ makes a second jump, which depends in general on the conditions at the end point of the first jump.

- $q_{i j}(i, j=1,2, \ldots, n)$ is the probability that an individual arrives at patch $j$ by a jump from patch $i$. Therefore, $Q=\left(q_{i j}\right)$ is an $n \times n$ matrix describing 
the instantaneous spatial redistribution of migrating individuals. Note that this matrix characterizes the spatial structure, which will be assumed to be identical for all species. We shall discuss this matrix in detail in the next section.

It is clear, when focussing on a certain patch $j$, that the term $M u_{j}$ is the emigration rate, whereas the term $\left(I-\Phi\left(u_{j}\right)\right) \sum_{k=1}^{n} q_{k j} M u_{k}$ is the immigration rate via a single jump and the term $\sum_{l=1}^{n} q_{l j} \Phi\left(u_{l}\right) \sum_{k=1}^{n} q_{k l} M u_{k}$ is the immigration rate via double jumps.

If we define a new matrix $C=\left(c_{i j}\right)_{n \times n} \equiv Q-I$ in which $I$ is the $n \times n$ identity matrix, i.e.,

$$
c_{i j}= \begin{cases}q_{i j} & \text { if } i \neq j \\ q_{i j}-1 & \text { if } i=j,\end{cases}
$$

(2.1) can be rewritten as

$$
\begin{gathered}
\frac{d u_{j}}{d t}=f\left(u_{j}\right)+\sum_{k=1}^{n} c_{k j} M u_{k}+\sum_{l=1}^{n} c_{l j} \Phi\left(u_{l}\right) \sum_{k=1}^{n} q_{k l} M u_{k} \\
j=1,2, \ldots, n .
\end{gathered}
$$

We call (2.3) the standard or single-jump equation when $\boldsymbol{\Phi}=\mathbf{0}$, and the doublejump equation when $\boldsymbol{\Phi} \neq \mathbf{0}$.

\section{JUMP REDISTRIBUTION}

The matrix $Q$ describing the instantaneous jumps is one of the important components of the model. Since the elements of $Q$ are probabilities we require

$$
0 \leq q_{i j} \leq 1 \quad \text { for } i, j=1,2, \ldots, n .
$$

We neglect the possibility of death during an instantaneous jump, and accordingly assume that the conservation relation

$$
\sum_{j=1}^{n} q_{i j}=1 \quad \text { for } i=1,2, \ldots, n
$$

holds. In addition, we restrict ourselves to the case of nondirectional dispersal (e.g., governed only by the distance):

$$
q_{i j}=q_{j i} \quad \text { for } i, j=1,2, \ldots, n \text {. }
$$


Therefore, $Q$ is diagonalizable and it has $n$ real eigenvalues, one of which is one, the others being smaller than one. Indeed, all eigenvalues of $Q$ lie in $[-1,1]$ as follows from the well-known Gerschgorin Theorem in matrix theory [see e.g., Ortega (1987)] (see the Appendix for two examples of the matrix). Immediately one finds that the matrix $C=Q-I$ is diagonalizable as well and it has $n$ real eigenvalues, one of which is zero, the others are negative, lying in $[-2,0)$. We shall denote the eigenvalues of $C$ by $\lambda_{i}, i=1,2, \ldots, n$ and order them such that

$$
0=\lambda_{1}>\lambda_{2} \geq \cdots \geq \lambda_{n} \geq-2 \text {. }
$$

\section{Stability Criteria of the flat Solutions}

It is clear that the assumptions about the matrix $Q$ are such that the system (2.3) allows spatially homogeneous (or, flat) solutions. A flat solution has the form

$$
S(t)=(s(t), \ldots, s(t)) \in \mathbb{R}^{m \times n}
$$

where $s(t) \in \mathbb{R}^{m}$ is the solution to the one-patch problem, i.e.,

$$
\dot{s}=f(s) .
$$

The linear stability analysis of a flat solution to the $n$-patch system (2.3) can be simplified, as detailed in the following result.

THEOREM 4.1. The linearization of (2.3) around a flat solution $S(t)=(s(t), \ldots$, $s(t))$ can be transformed by a similarity transformation into $n$ decoupled subsystems

$$
\dot{w}_{j}=\left\{D f(s)+\lambda_{j} M\left[I+H(s)+\left(1+\lambda_{j}\right) \Phi(s)\right]\right\} w_{j}, \quad j=1,2, \ldots, n .
$$

Here $H(s)=\operatorname{diag}\left\{s_{1}, \ldots, s_{m}\right\} D \phi(s)$.

Remark. This theorem extends the previous results about the stability analysis of flat solutions in multi-patch models (Othmer and Scriven, 1971; Jansen and Lloyd, 2000; Huang and Diekmann, 2003); a flat solution of (2.3) is linearly stable if and only if the zero solutions of the $n$ subsystems given by (4.1) are all asymptotically stable. Hereafter, we shall call the matrices $B_{j}$ defined by

$$
B_{j}=D f(s)+\lambda_{j} M\left[I+H(s)+\left(1+\lambda_{j}\right) \Phi(s)\right], \quad j=1,2, \ldots, n
$$

as the criterion matrices. When $s(t)=s$ is an equilibrium, the flat solution $S=(s, \ldots, s)$ is linearly stable if all eigenvalues of the criterion matrices $B_{j}$ have negative real parts. When $s(t+T)=s(t)$, i.e., $s(t)$ is periodic with period $T$, the flat solution $S(t)$ is linearly stable if all nontrivial Floquet multipliers of the system $\dot{w}_{j}=B_{j}(s(t)) w_{j}$ for all $j$ lie inside the unit circle. 
Proof of Theorem 4.1. For any solution $\left(u_{1}, \ldots, u_{n}\right)$ and a flat solution $(s, \cdot, s)$ of (2.3) we can write

$$
\begin{aligned}
\dot{u}_{j}-\dot{s}= & f\left(u_{j}\right)-f(s)+\sum_{k=1}^{n} c_{k j} M\left(u_{k}-s\right) \\
& +\sum_{l=1}^{n} c_{l j} \sum_{k=1}^{n} q_{k l} M\left[\Phi\left(u_{l}\right) u_{k}-\Phi(s) s\right] \quad j=1,2, \ldots, n
\end{aligned}
$$

The following Taylor expansion holds for any pair of $m$-dimensional vectors $x=$ $\left(x_{1}, \ldots, x_{m}\right) \in \mathbb{R}^{m}$ and $y=\left(y_{1}, \ldots, y_{m}\right) \in \mathbb{R}^{m}$ with both $x-s$ and $y-s$ small:

$$
\Phi(x) y=\Phi(s) s+H(s)(x-s)+\Phi(s)(y-s)+\text { h.o.t. }
$$

in which

$$
H(s)=\operatorname{diag}\left\{s_{1}, \ldots, s_{m}\right\} D \phi(s):=\left.\left(\begin{array}{ccc}
\frac{\partial \phi_{1}(x)}{\partial x_{1}} y_{1} & \ldots & \frac{\partial \phi_{1}(x)}{\partial x_{m}} y_{1} \\
\ldots & \ldots & \cdots \\
\frac{\partial \phi_{m}(x)}{\partial x_{1}} y_{m} & \cdots & \frac{\partial \phi_{m}(x)}{\partial x_{m}} y_{m}
\end{array}\right)\right|_{x=y=s} .
$$

Let

$$
u_{j}(t)=z_{j}(t)+s(t), \quad j=1,2, \ldots, n
$$

with $z_{j}(t)$ small. Then we find, for any $l, k=1,2, \ldots, n$, that

$$
\Phi\left(u_{l}\right) u_{k}-\Phi(s) s=H(s) z_{l}+\Phi(s) z_{k}+\text { h.o.t. }
$$

The dynamics of $z_{j}(t), j=1,2, \ldots, n$ can therefore be approximated by the linear system

$$
\begin{aligned}
\dot{z}_{j}= & D f(s) z_{j}+\sum_{k=1}^{n} c_{k j} M z_{k} \\
& +\sum_{l=1}^{n} c_{l j} \sum_{k=1}^{n} q_{k l} M\left[H(s) z_{l}+\Phi(s) z_{k}\right], \quad j=1,2, \ldots, n .
\end{aligned}
$$

Since $\sum_{k=1}^{n} q_{k l}=1$ [which follows from (3.2) and (3.3)], we find that

$$
\begin{aligned}
\dot{z}_{j}= & D f(s) z_{j}+\sum_{k=1}^{n} c_{k j} M(I+H(s)) z_{k} \\
& +\sum_{l=1}^{n} c_{l j} \sum_{k=1}^{n} q_{k l} M \Phi(s) z_{k}, \quad j=1,2, \ldots, n .
\end{aligned}
$$


To perform a linear transformation, we write the above system in the following matrix form

$$
\begin{aligned}
\left(\dot{z}_{1}, \ldots, \dot{z}_{n}\right)= & \left(D f(s) z_{1}, \ldots, D f(s) z_{n}\right) \\
& +\left(M(I+H(s)) z_{1}, \ldots, M(I+H(s)) z_{n}\right) C \\
& +\left(\sum_{k=1}^{n} q_{k l} M \Phi(s) z_{k}, \ldots, \sum_{k=1}^{n} q_{k n} M \Phi(s) z_{k}\right) C
\end{aligned}
$$

which can be further elaborated, using $Q=C+I$, as

$$
\begin{aligned}
\left(\dot{z}_{1}, \ldots, \dot{z}_{n}\right)= & \left(D f(s) z_{1}, \ldots, D f(s) z_{n}\right) \\
& +\left(M(I+H(s)+\Phi(s)) z_{1}, \ldots, M(I+H(s)+\Phi(s)) z_{n}\right) C \\
& +\left(M \Phi(s) z_{1}, \ldots, M \Phi(s) z_{n}\right) C^{2} .
\end{aligned}
$$

Because $C$ is similar to the diagonal matrix $\Lambda=\operatorname{diag}\left\{\lambda_{1}, \ldots, \lambda_{n}\right\}$ in which $\lambda_{1}, \ldots, \lambda_{n}$ are the $n$ eigenvalues of $C$, there exists a nonsingular $n \times n$ matrix $P$ such that

$$
P^{-1} C P=\Lambda=\operatorname{diag}\left\{\lambda_{1}, \ldots, \lambda_{n}\right\} \quad \text { or } \quad C P=P \Lambda .
$$

Multiplying (4.10) from the right by $P$ and using the similarity transformation

$$
w_{j}=z_{j} P, \quad j=1,2, \ldots, n
$$

we obtain

$$
\begin{aligned}
\left(\dot{w}_{1}, \ldots, \dot{w}_{n}\right)= & \left(D f(s) w_{1}, \ldots, D f(s) w_{n}\right) \\
& +\left(M[I+H(s)+\Phi(s)] w_{1}, \ldots, M[I+H(s)+\Phi(s)] w_{n}\right) \Lambda \\
& +\left(M \Phi(s) w_{1}, \ldots, M \Phi(s) w_{n}\right) \Lambda^{2}
\end{aligned}
$$

which consists of $n$ decoupled $m$-dimensional subsystems

$$
\dot{w}_{j}=\left\{D f(s)+\lambda_{j} M\left[I+H(s)+\Phi(s)+\lambda_{j} \Phi(s)\right]\right\} w_{j}, \quad j=1,2, \ldots, n .
$$




\section{EFFECTS ON Diffusive InSTABILITY}

Throughout this section we restrict ourselves to the two species case (i.e., $m=2)$. Let $S=(s, \ldots, s)$ be a homogeneous equilibrium of (2.3) in which $s=\left(s_{1}, s_{2}\right)^{T}$ is a stable equilibrium of the one-patch problem $\dot{u}=f(u)$. For the standard equation $(\boldsymbol{\Phi}=\mathbf{0})$ the criterion matrices are

$$
B_{j}=D f(s)+\lambda_{j} M, \quad j=1,2, \ldots, n
$$

whereas for the double-jump equation $(\boldsymbol{\Phi} \neq \mathbf{0})$ the criterion matrices are

$$
B_{j}=D f(s)+\lambda_{j}\left[I+H(s)+\left(\lambda_{j}+1\right) \Phi(s)\right] M, \quad j=1,2, \ldots, n .
$$

Let $D f(s)=\left(f_{i j}\right)_{2 \times 2}$. The assumption that the equilibrium of the one-patch problem $s$ is linearly stable implies that

$$
\begin{cases}f_{11}+f_{22} & <0 \\ f_{11} f_{22}-f_{12} f_{21} & >0\end{cases}
$$

Let $D \phi(s)=\left(\phi_{i j}\right)_{2 \times 2}$. We then find, by definition, that

$$
H(s)=\operatorname{diag}\left\{s_{1}, s_{2}\right\} D \phi(s)=\left(\begin{array}{ll}
s_{1} \phi_{11} & s_{1} \phi_{12} \\
s_{2} \phi_{21} & s_{2} \phi_{22}
\end{array}\right) .
$$

It follows that the criterion matrix $B_{j}$ (for $\lambda_{j} \in[-2,0]$ and $\phi_{1}, \phi_{2} \in[0,1]$ ) has the form

in which

$$
B\left(\lambda_{j}, \phi_{1}, \phi_{2}, \alpha, \beta\right)=\left(\begin{array}{cc}
f_{11}+\lambda_{j} \hat{m}_{1} & f_{12}+\lambda_{j} \alpha \\
f_{21}+\lambda_{j} \beta & f_{22}+\lambda_{j} \hat{m}_{2}
\end{array}\right)
$$

$$
\begin{gathered}
\hat{m}_{i}=m_{i}\left[1+\left(\lambda_{j}+1\right) \phi_{i}+s_{i} \phi_{i i}\right], \quad i=1,2 \\
\alpha=s_{1} \phi_{12} \quad \beta=s_{2} \phi_{21} .
\end{gathered}
$$

We shall assume that $\phi_{i i} \geq 0$ for $i=1,2$. It follows that $\hat{m}_{i} \geq 0(i=1,2)$ for any $\lambda_{j} \in[-2,0]$ and $\phi_{1}, \phi_{2} \in[0,1]$.

$S$ is stable if and only if

$$
\begin{cases}f_{11}+f_{22}+\lambda_{j}\left(\hat{m}_{1}+\hat{m}_{2}\right) & <0 \\ K\left(\lambda_{j}, \phi_{1}, \phi_{2}, \alpha, \beta\right) & >0\end{cases}
$$

in which

$$
\begin{aligned}
K\left(\lambda_{j}, \phi_{1}, \phi_{2}, \alpha, \beta\right)= & f_{11} f_{22}-f_{12} f_{21}+\lambda_{j}\left(f_{11} \hat{m}_{2}+f_{22} \hat{m}_{1}\right)+\lambda_{j}^{2} \hat{m}_{1} \hat{m}_{2} \\
& -\lambda_{j} f_{21} \alpha-\lambda_{j} f_{12} \beta-\lambda_{j}^{2} \alpha \beta .
\end{aligned}
$$

Since the first inequality in (5.8) holds for any $\lambda_{j} \in[-2,0]$ under the assumption (5.3) (note that $m_{1}, m_{2}$ are nonnegative and that $\phi_{1}, \phi_{2} \in[0,1]$ ), the diffusive instability arises if and only if $K\left(\lambda_{j}, \phi_{1}, \phi_{2}, \alpha, \beta\right)<0$ for some $\lambda_{j} \in[-2,0)$. 
5.1. Density-independent second jump. Let us look in this subsection at the case where $\Phi$ is density-independent. In this case $\boldsymbol{H}(\boldsymbol{s})=\mathbf{0}$. It follows that $\alpha=\beta=0$ and that

$$
\begin{aligned}
K\left(\lambda_{j}, \phi_{1}, \phi_{2}, 0,0\right)= & f_{11} f_{22}-f_{12} f_{21}+\lambda_{j}\left[1+\left(\lambda_{j}+1\right) \phi_{1}\right] f_{22} m_{1} \\
& +\lambda_{j}\left[1+\left(\lambda_{j}+1\right) \phi_{2}\right] f_{11} m_{2} \\
& +\lambda_{j}^{2}\left[1+\left(\lambda_{j}+1\right) \phi_{1}\right]\left[1+\left(\lambda_{j}+1\right) \phi_{2}\right] m_{1} m_{2} .
\end{aligned}
$$

For competitive interaction, it is natural that $f_{12}<0, f_{21}<0$. It follows from the general assumption (5.3) that $f_{11}<0, f_{22}<0$. Therefore, $K\left(\lambda_{j}, \phi_{1}, \phi_{2}, 0,0\right)>0$ for all $\lambda_{j} \in[-2,0]$, which suggests that the system (2.3) cannot exhibit diffusive instability.

For prey-predator (or activator-inhibitor) interaction it is natural that $f_{12}$ and $f_{21}$ have the opposite signs. It follows from the general assumption (5.3) that $f_{11} f_{22}<0$. Suppose, without loss of generality, that $f_{11}>0, f_{22}<0$. The neutral stability condition $K\left(\lambda_{j}, \phi_{1}, \phi_{2}, 0,0\right)=0$ can then be written as

$$
m_{2}=\frac{\left(f_{11} f_{22}-f_{12} f_{21}\right)+f_{22} \lambda_{j}\left[1+\left(\lambda_{j}+1\right) \phi_{1}\right] m_{1}}{-\lambda_{j}\left[1+\left(\lambda_{j}+1\right) \phi_{2}\right]\left\{f_{11}+\lambda_{j}\left[1+\left(\lambda_{j}+1\right) \phi_{1}\right] m_{1}\right\}}
$$

which defines a curve in the $\left(m_{1}, m_{2}\right)$ plane (while $\left.0 \leq m_{1} \leq-\frac{f_{11}}{\lambda_{j}\left[1+\left(\lambda_{j}+1\right) \phi_{1}\right]}\right)$ when the other parameters are fixed. It is clear that the homogeneous equilibrium $S$ becomes unstable in the $j$ th eigenmode as the point $\left(m_{1}, m_{2}\right)$ moves upwards over the curve. Some simple algebra shows that the curve moves upwards as $\phi_{2}$ increases from 0 to 1 for any $\left|\lambda_{j}\right| \leq 1$ (while the other parameters are fixed) and that the curve moves downwards as $\phi_{2}$ increases from 0 to 1 for any $\left|\lambda_{j}\right|>1$ (while the other parameters are fixed) (see the two upper pictures in Fig. 1 where we plot the curve for the two extremes of $\phi_{2}$ only). It can be shown similarly that the curve moves to the left as $\phi_{1}$ increases for any $\left|\lambda_{j}\right| \leq 1$ and that the curve moves to the right as $\phi_{1}$ increases for any $\left|\lambda_{j}\right|>1$ (see the two lower pictures in Fig. 1 where we plot the curve for the two extremes of $\phi_{1}$ only). In summary, we have

Proposition 5.1. Without density-dependent mechanisms in making jumps both the double-jump equation and the standard equation exhibit no diffusive instability for competitive interactions. For predator-prey (or activator-inhibitor) interactions, both equations could exhibit diffusive instability, for which the conditions differ quantitatively.

5.2. Density-dependent second jump. In this subsection we consider the case where one or both diagonal elements of $\Phi$ are density-dependent. We shall speak of the cross-response second jump if the probability that individuals of species $i$ make a second jump (i.e., $\phi_{i}$ ) depends on the density of the other species. Now, one 

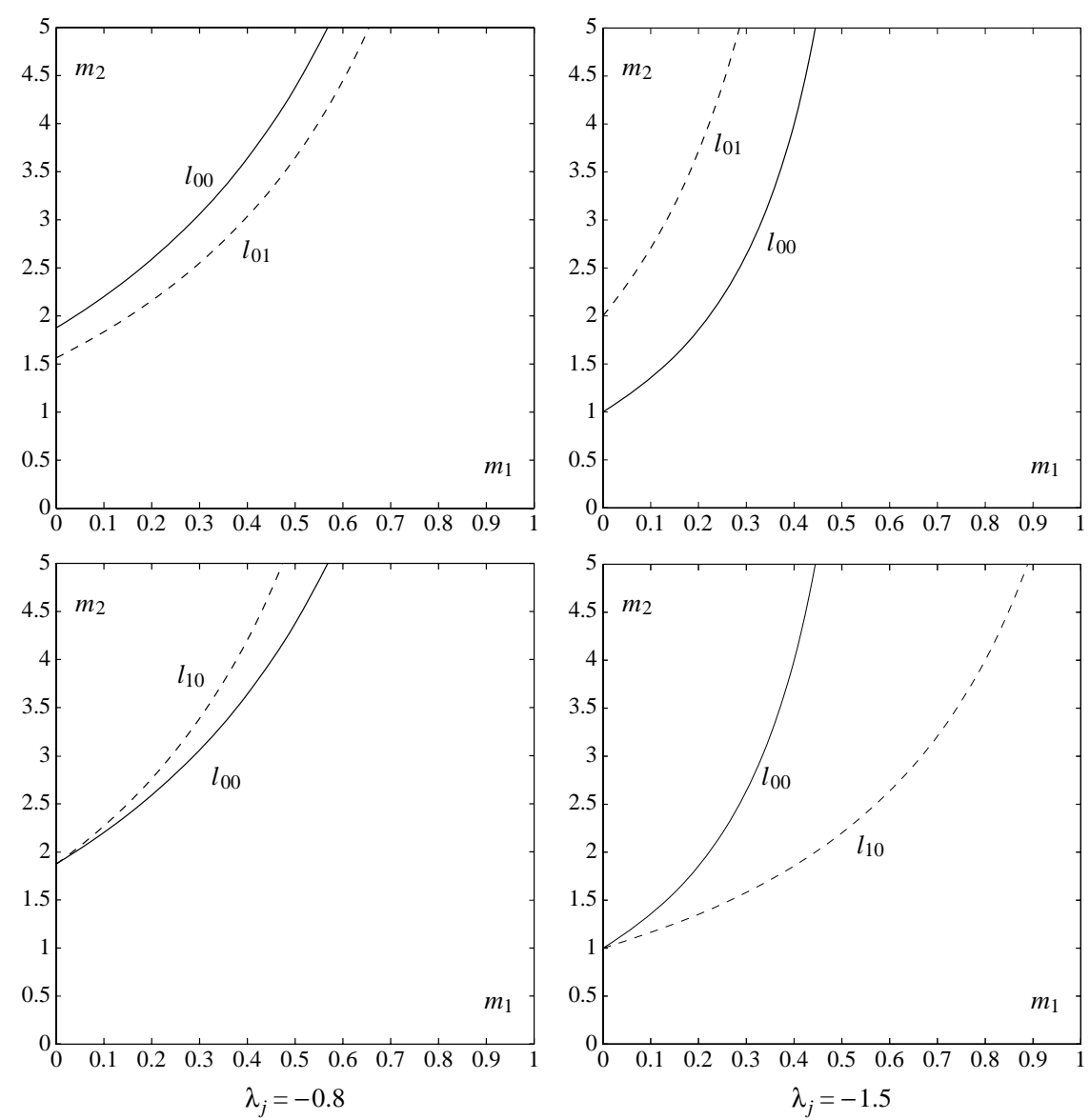

Figure 1. Neutral stability curves $\left(l_{i j}\right.$ corresponds to the neutral stability condition $\operatorname{Det}\left(B\left(\lambda_{j}, \phi_{1}, \phi_{2}, 0,0\right)\right)=0$ for $\left.\phi_{1}=i, \phi_{2}=j, i, j=0,1\right)$. The homogeneous equilibrium becomes linearly unstable in the $j$ th eigenmode of the linearized system when the point $\left(m_{1}, m_{2}\right)$ moves upwards over a curve in each picture. The other fixed parameters are: $f_{11}=1, f_{22}=-1.5, f_{12}=-2, f_{21}=1.5$.

finds that $H(s)=\operatorname{diag}\left\{s_{1}, \ldots, s_{m}\right\} D \phi(s)$ is a full matrix (rather than a diagonal matrix) which means that $\alpha \neq 0, \beta \neq 0$. Note that $|\alpha|$ and $|\beta|$ measure the strength of the cross-response of the second jump.

For competitive interaction, $f_{12}, f_{21}$ and $\alpha, \beta$ should satisfy

$$
f_{12}<0, \quad f_{21}<0, \quad \alpha \geq 0, \quad \beta \geq 0
$$

where the positive sign of $\alpha$ and $\beta$ means that the inclination of individuals of one species to make a second jump increases as the number of the competitors increases.

Checking the expression of the function $K$ defined in (5.9) we find that all terms involving $\alpha$ and/or $\beta$ are negative when $\alpha \neq 0, \beta \neq 0$ according to the sign 
assumption (5.12) and that the sum of these terms decreases at a rate bounded away from zero as $\alpha$ and/or $\beta$ increase. Therefore the function $K$ shall be negative sooner or later as $\alpha$ and/or $\beta$ increase. In other words, the diffusive instability shall arise when the cross-response (of the second jump) is sufficiently strong.

For prey-predator (or activator-inhibitor) interaction, if the prey and the predator correspond to, respectively, the indices 1 and 2, we should have

$$
f_{12}<0, \quad f_{21}>0, \quad \alpha \geq 0, \quad \beta \leq 0
$$

where the positive sign of $\alpha$ implies that the inclination of prey to make a second jump increases as the number of predators increases, whereas the negative sign of $\beta$ suggests that the tendency of predators to make a second jump decreases as the number of prey increases.

For this type of interaction, $S$ may be diffusively stable or unstable when $\alpha=$ $\beta=0$, depending on the precise local interaction terms. Let us first assume that $S$ is diffusively stable when $\alpha=\beta=0$, namely

$$
K\left(\lambda_{j}, \phi_{1}, \phi_{2}, 0,0\right)>0, \quad \text { for all } \lambda_{j} \in[-2,0) .
$$

Then we find, according to the sign assumptions on $f_{12}, f_{21}$ and $\alpha, \beta$, that

$$
K\left(\lambda_{j}, \phi_{1}, \phi_{2}, \alpha, \beta\right)>0, \quad \text { for all } \lambda_{j} \in[-2,0)
$$

as well as when $\alpha \neq 0$ and/or $\beta \neq 0$ [because all terms involving $\alpha$ and/or $\beta$ are positive when $\alpha \neq 0, \beta \neq 0$ according to the sign assumption (5.13)]. Therefore, the equilibrium $S$ can not be destabilized by the cross-response (of the second jump).

Next, we assume that $S$ is diffusively unstable when $\alpha=\beta=0$, namely

$$
K\left(\lambda_{j}, \phi_{1}, \phi_{2}, 0,0\right)<0, \quad \text { for some } \lambda_{j} \in[-2,0) .
$$

Now, the fact that all terms involving $\alpha$ and/or $\beta$ in the expression of the function $K$ are positive and that the sum of these terms increases at a rate bounded away from zero as $\alpha$ and/or $|\beta|$ increase means that the function $K$ shall be positive sooner or later as $\alpha$ and/or $|\beta|$ increase. In other words, the equilibrium $S$ shall be stable when the cross-response (of the second jump) is sufficiently strong. In summary, we have

Proposition 5.2. For a competitive type of interaction (or mutualism), a standard equation does not exhibit diffusive instability, but a cross-response second jump may lead to diffusive instability provided that the response is sufficiently strong. For a prey-predator (or an activator-inhibitor) type of interaction, a standard equation may be either stable or unstable. A cross-response second jump can stabilize an unstable standard equation provided that the response is sufficiently strong, but it never destabilizes a stable standard equation. 


\section{EXAMPLES}

6.1. Competition. Let us consider a multi-patch two-species competitive system [formulated as (2.3)] in which the identical local dynamics are described by the following Lotka-Volterra equations

$$
\begin{aligned}
\dot{u} & =u\left(a_{1}-b_{1} u-c_{1} v\right) \\
\dot{v} & =v\left(a_{2}-b_{2} u-c_{2} v\right)
\end{aligned}
$$

where all the constants $a_{i}, b_{i}, c_{i}, i=1,2$ are positive. $u$ and $v$ are respectively the population densities of the two competing species. In the 'weak competition' case, i.e.,

$$
\frac{b_{1}}{b_{2}}>\frac{a_{1}}{a_{2}}>\frac{c_{1}}{c_{2}}
$$

(6.1) has a positive steady state

$$
\left(u^{*}, v^{*}\right)=\left(\frac{a_{1} c_{2}-a_{2} c_{1}}{b_{1} c_{2}-b_{2} c_{1}}, \frac{b_{1} a_{2}-b_{2} a_{1}}{b_{1} c_{2}-b_{2} c_{1}}\right) .
$$

Standard equation. The corresponding equation is (2.3) with $\boldsymbol{\Phi}=\mathbf{0}$. It is wellknown that the homogeneous steady state of $(2.3)$, i.e., $S=(s, \ldots, s)$ with $s=$ $\left(u^{*}, v^{*}\right)^{T}$, is globally asymptotically stable no matter how large the constants $m_{1}$ and $m_{2}$ are.

Double-jump equation. The corresponding equation is (2.3) with $\boldsymbol{\Phi} \neq \mathbf{0}$. Let us assume that the probability that individuals of species 1 make a second jump is constant and that the probability that individuals of species 2 make a second jump depends only on the density of species 1 , i.e., $\Phi=\operatorname{diag}\left\{\phi_{1}, \phi_{2}(u)\right\}$. To show that a strong second-jump response does lead to diffusive instability, $\phi_{2}(u)$ has to be of the right functional form. Suppose that it is given as the Hill function, i.e.,

$$
\phi_{2}(u)=\frac{u^{\theta}}{\left(u^{*}\right)^{\theta}+u^{\theta}}, \quad u \geq 0, \theta \geq 0
$$

[see Fig. 2 (the left picture) for its graphs for the various values of $\theta$ ], one then easily computes that

$$
\beta=\left.\left[\phi_{2}^{\prime}(u) v\right]\right|_{u=u^{*}, v=v^{*}}=\phi_{2}^{\prime}\left(u^{*}\right) v^{*}=\frac{v^{*}}{4 u^{*}} \theta
$$

which implies that the strength of the second-jump response of species 2 to the density of species 1 is measured by the parameter $\theta$.

By Proposition 5.2, $S$ becomes unstable when $\theta$ is sufficiently large (see Fig. 3). 

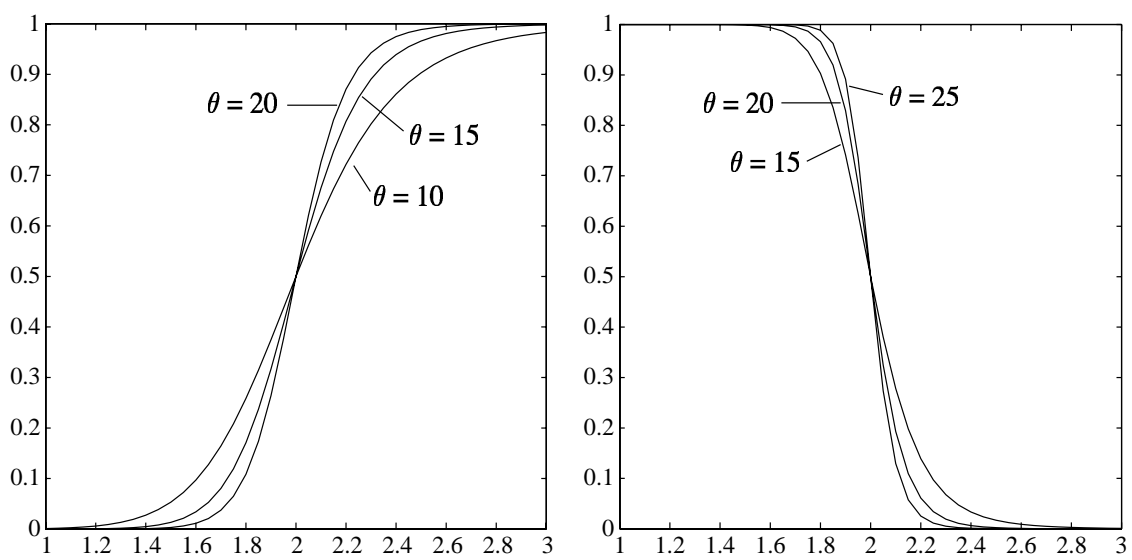

Figure 2. Left picture: the graphs of the function $\phi(x)=\frac{x^{\theta}}{x^{\theta}+2^{\theta}}$ for the various values of $\theta$. Right picture: the graphs of the function $\phi(x)=\frac{2^{\theta}}{x^{\theta}+2^{\theta}}$ for the various values of $\theta$.

6.2. Predator-prey interaction. Let us consider a multi-patch phytoplanktonherbivore system [formulated as (2.3)] in which the identical local dynamics are determined by the equations (Levin and Segel, 1976)

$$
\begin{aligned}
& \dot{v}=a v+e v^{2}-b_{1} v p \\
& \dot{p}=-c p^{2}+b_{2} v p
\end{aligned}
$$

which reflect the assumptions that there is an autocatalytic effect in the growth rate of phytoplankton $(v)$ and that the mortality of the herbivore $(p)$ is densitydependent. All parameters $a, b_{1}, b_{2}, c$ and $e$ are positive.

When $b_{1} b_{2}>c e$ and $b_{2}>e$, (6.4) has an asymptotically stable steady state

$$
\left(v^{*}, p^{*}\right)=\left(\frac{a c}{b_{1} b_{2}-c e}, \frac{a b_{2}}{b_{1} b_{2}-c e}\right) .
$$

Standard equation. The corresponding equation is (2.3) with $\mathbf{\Phi}=\mathbf{0}$. It is known that when

$$
\frac{m_{2}}{m_{1}}>\frac{1}{\left[\sqrt{b_{1} / c}-\sqrt{b_{1} / c-e / b_{2}}\right]^{2}}
$$

diffusive instability occurs.

Double-jump equation. The corresponding equation is (2.3) with $\boldsymbol{\Phi} \neq \mathbf{0}$. Let us assume that the probability that individuals of species 1 make a second jump is constant and that the probability that individuals of species 2 make a second jump depends only on the density of species 1, i.e., $\Phi=\operatorname{diag}\left\{\phi_{1}, \phi_{2}(u)\right\}$. Suppose that 


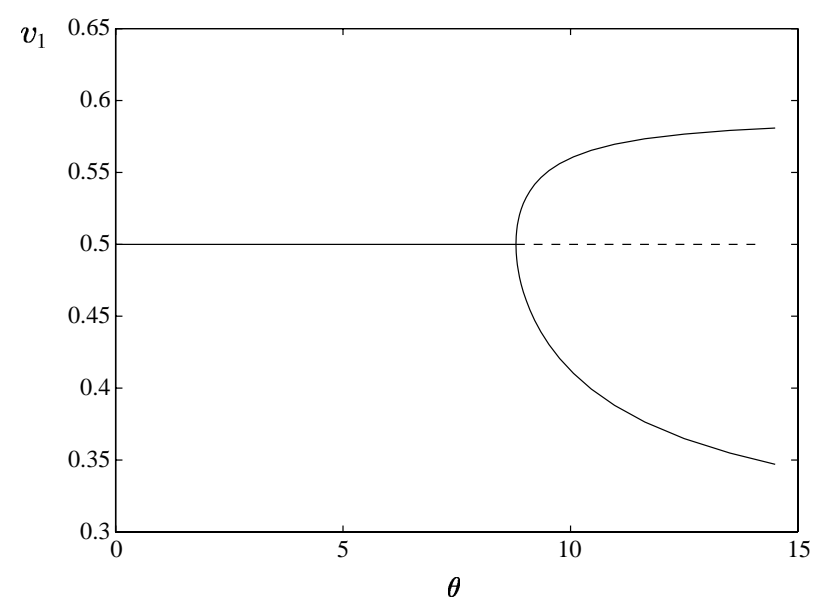

Figure 3. A one-parameter bifurcation diagram of the two-patch competition system with a double-jump scheme of migration. The local dynamics are described by (6.1). $\phi_{1}=1$, $\phi_{2}$ is given by (6.3). It is clear that inhomogeneous equilibria arise as the parameter $\theta$, which measures the strength of the second-jump response of the species 2 to the density of species 1 , increases. (Note that $v_{1}$ in the figure is the prey density in patch 1 .) The other parameters are $a_{1}=b_{1}=c_{1}=1, a_{2}=2, b_{2}=1, c_{2}=3 ; m_{1}=0.1, m_{2}=2.5$; the redistribution matrix $Q$ is set to be $q_{11}=q_{12}=q_{21}=q_{22}=0.5$.

$$
\phi_{2}(v)=\frac{\left(v^{*}\right)^{\theta}}{\left(v^{*}\right)^{\theta}+v^{\theta}}, \quad u \geq 0, \theta \geq 0
$$

[see Fig. 2 (the right picture) for its graphs for the various values of $\theta$ ]. One can easily compute that

$$
\beta=\left.\left[\phi_{2}^{\prime}(v) p\right]\right|_{v=v^{*}, p=p^{*}}=\phi_{2}^{\prime}\left(v^{*}\right) p^{*}=-\frac{p^{*}}{4 v^{*}} \theta .
$$

So the parameter $\theta$ measures the strength of the second-jump response of predators to the density of prey.

Thus, diffusive instability never occurs when $\theta$ is sufficiently large according to Proposition 5.2.

\section{Discussion}

The migration process of some species might be so complicated that it cannot be considered as a simple Markov process, as has been the standard approach to describe the migration of organisms. In this paper we considered the case where a migration event consists of one or two instantaneous jumps (by a jump we mean an instantaneous take-off-and-landing process). It turned out that a densityindependent second jump has some quantitative effects on diffusive instability. When a second jump happens as a natural interspecific response of individuals, and such a response is sufficiently strong, it has crucial effects on diffusive instability: 
it leads to diffusive instability in the case of competitive interactions, whereas it annihilates diffusive instability in the case of prey-predator interactions. So the present results reinforce our earlier (Huang and Diekmann, 2003) conclusion that interspecific influence on mobility crucially affects diffusive instability.

In the case of two-species competition, the local growth rate of one species decreases as the abundance of the competitive species increases, while the inclination of individuals of the species to make a second jump increases as the abundance of the competitive species increases. In the case of predator-prey interactions, the local growth rate of the predator increases as the abundance of the prey increases, while the inclination of predators to make a second jump decreases as the abundance of prey increases. So there is a natural relation between the local interaction and the second-jump response in each type of interaction. This relation leads to our results.

Neubert et al. (2002) have considered a multi-patch predator-prey model in which individual travel times as well as deaths during travel are explicitly incorporated in the form of dispersal delays and showed that such dispersal delays are almost always stabilizing. Othmer et al. (1988) have characterized a dispersal process consisting of a number of jumps where the waiting times between jumps are explicitly considered. For the migration process we considered in this paper, the assessment before deciding whether or not to make a second jump might take time. Such a time could be incorporated into the model in terms of a time delay. Doing so, however, would extremely decrease the mathematical tractability of the model. We therefore did not consider this situation in this paper. We focussed on the stability consequence of the density-dependence factor (rather than that of the time delays).

Both the continuous- and the discrete-time approaches can be used to describe the spatio-temporal change of population densities. For the discrete-time approach, the strength of migration is reflected by the proportion that individuals migrate per generation, whereas for the continuous-time approach, the strength of migration is reflected by the per capita rate at which individuals migrate. In order to study the effects of the (cross-) density-dependence at the end of a migration event on diffusive instability we formulated the continuous-time model with double-jump migration. Clearly one can also study the issue by discrete-time models which may be formulated by extending the standard models (e.g., Ruxton, 1996; Rohani and Ruxton, 1999; Silva et al., 2001).

We may extend the model (2.3) further to let $M$ be cross-density-dependent. Namely, we may further consider the case where both a cross-emigration response and a cross-second-jump response exist. One can check that the computation procedure in Section 5 carries over. The resulting model with both mechanisms (viz, both the cross-response of second jump and the cross-response of emigration) hence should exhibit diffusive instability easier in the case of competition, but more difficult in the case of predator-prey interactions as compared to the model with only one mechanism. 


\section{ACKNOWLEDGements}

We thank two anonymous referees for their comments leading to several improvements in the exposition.

\section{Appendix A: Examples Of The Matrix $Q$}

In the case that $n$ patches are arranged in a one-dimensional linear chain, $Q$ may have, for instance, the form

$$
Q=\left[\begin{array}{ccccccc}
\frac{1}{2} & \frac{1}{2} & 0 & \cdots & & \cdots & 0 \\
\frac{1}{2} & 0 & \frac{1}{2} & \cdots & & \cdots & 0 \\
0 & \frac{1}{2} & 0 & \frac{1}{2} & & \cdots & 0 \\
\vdots & \vdots & \vdots & \vdots & \vdots & \vdots & \vdots \\
0 & \cdots & & 0 & \frac{1}{2} & 0 & \frac{1}{2} \\
0 & \cdots & & \cdots & 0 & \frac{1}{2} & \frac{1}{2}
\end{array}\right] .
$$

The $n$ eigenvalues are $\sigma_{i}=\cos \frac{i-1}{n} \pi, i=1, \ldots, n$, which lie in $[-1,1]$. It follows that the $n$ eigenvalues of $C=Q-I$ are $\lambda_{i}=-1+\cos \frac{i-1}{n} \pi, i=1, \ldots, n$. These eigenvalues lie in $[-2,0]$.

In the case that $n$ patches $(n \geq 3)$ form a ring, $Q$ typically is a circulant matrix. For instance

$$
Q=\left[\begin{array}{ccccccc}
a & b & 0 & \cdots & & 0 & b \\
b & a & b & \cdots & & \cdots & 0 \\
0 & b & a & b & & \cdots & 0 \\
\vdots & \vdots & \vdots & \vdots & \vdots & \vdots & \vdots \\
0 & \cdots & & 0 & b & a & b \\
b & 0 & & \cdots & 0 & b & a
\end{array}\right]
$$

where $a, b>0$ and $a+2 b=1$. The $n$ eigenvalues are $\sigma_{i}=a+2 b \cos \frac{2(i-1)}{n} \pi, i=$ $1, \ldots, n$, which lie in $[-1,1]$. It follows that the $n$ eigenvalues of $C=Q-I$ are $\lambda_{i}=-1+a+2 b \cos \frac{2(i-1)}{n} \pi=-2 b+2 b \cos \frac{2(i-1)}{n} \pi, i=1, \ldots, n$. These eigenvalues belong to $[-2,0]$.

\section{REFERENCES}

Aronson, D. G. (1985). The role of diffusion in mathematical population biology: Skellam revisited, In Mathematics in Biology and Medicine, V. Capasso, (Ed.), Lecture Notes in Biomathematics 57, pp 2-6.

Diekmann, O. (1978). Thresholds and traveling waves for the geographical spread of infection. J. Math. Biol. 6, 109-130. 
Diekmann, O. (1979). Run for your life: a note on the asymptotic speed of propagation of an epidemic. J. Differ. Equ. 33, 58-73.

Holt, R. D. (1984). Spatial heterogeneity, indirect interaction, and the coexistence of prey species. Am. Nat. 124, 377-406.

Holt, R. D. (1985). Population dynamics in two-patch environments: some anomalous consequences of an optimal habitat distribution. Theor. Popul. Biol. 28, 181-208.

Huang, Y. and O. Diekmann (2001). Predator migration in response to prey density: what are the consequences?. J. Math. Biol. 43, 561-581.

Huang, Y. and O. Diekmann (2003). Interspecific influence on mobility and Turing instability. Bull. Math. Biol. 46, 143-156.

Jansen, V. A. A. and A. L. Lloyd (2000). Local stability analysis of spatially homogeneous solutions of multi-patch systems. J. Math. Biol. 41, 232-252.

Karevia, P. and P. Odell (1987). Swarms of predators exhibit preytaxis if individual predators use area restricted search. Am. Nat. 130, 233-270.

Kot, M. (1992). Discrete time travelling waves: ecological examples. J. Math. Biol. 30, 413-436.

Levin, S. A. and L. A. Segel (1976). Hypothesis for origin of planktonic patchiness. Nature 259, 659.

Lewis, M. A. and S. W. Pacala (2000). Modeling and analysis of stochastic invasion processes. J. Math. Biol. 41, 387-429.

Mollison, D. (1977). Spatial contact models for ecological and epidemic spread. J. R. Stat. Soc. B 39, 283-326.

Neubert, M. G., P. Klepac and P. van den Driessche (2002). Stabilizing dispersal delays in predato-prey metapopulation models. Theor. Popul. Biol. 61, 339-347.

Neubert, M. G., M. Kot and M. A. Lewis (1995). Dispersal and pattern formation in a discrete-time predator-prey model. Theor. Popul. Biol. 48, 7-43.

Okubo, A. (1980). Diffusion and Ecological Problems: Mathematical Models, Berlin: Springer.

Okubo, A. and S. A. Levin (2001). Diffusion and Ecological Problems: Modern Perspectives, 2nd edn, Berlin: Spinger.

Ortega, J. (1987). Matrix Theory, New York, London: Plenum Press.

Othmer, H. G., S. R. Dunbar and W. Alt (1988). Models of dispersal in biological systems. J. Math. Biol. 26, 263-298.

Othmer, H. G. and L. E. Scriven (1971). Instability and dynamic patterns in cellular networks. J. Theor. Biol. 32, 507-537.

Rohani, P. and G. D. Ruxton (1999). Dispersal-induced instabilities in host-parasitoid metapopulations. Theor. Popul. Biol. 55, 23-36.

Ruxton, G. D. (1996). Density-dependent migration and stability in a system of linked populations. Bull. Math. Biol. 58, 643-660.

Segel, L. A. (1984). Taxes in cellular ecology, In Mathematical Ecology, S. A. Levin, (Ed.), Lecture Notes in Biomathematics 54, pp. 407-424.

Silva, J. A. L., M. L. De Castro and D. A. R. Justo (2001). Stability in a metapopulation model with density-dependent dispersal. Bull. Math. Biol. 63, 485-505.

Skellam, J. G. (1973). The formulation and interpretation of mathematical models of diffusionary processes in population biology, in Mathematical Theory of Dynamics of Biological Populations, M. S. Barlett and R. W. Hiorns (Eds), New York: Academic Press, pp. 63-85. 
Thieme, H. R. (1979a). Asymptotic estimates of solutions of nonlinear integral equations and asymptotic speeds for the spread of populations. J. Reine Angew. Math. 306, 94-121.

Thieme, H. R. (1979b). Density-dependent regulation of spatially distributed populations and their asymptotic speed of spread. J. Math. Biol. 8, 173-187.

Turing, A. (1952). The chemical basis of morphogenesis. Phil. Trans. R. Soc. B. 237, 37-72.

Weinberger, H. F., M. A. Lewis and B. Li (2002). Analysis of linear determinacy for spread in cooperative models. J. Math. Biol. 45, 183-218.

Received 24 February 2003 and accepted 2 September 2003 